\title{
Impact of examined lymph node counts on survival of patients with stage IA non-small cell lung cancer undergoing sublobar resection
}

\author{
Yang Liu ${ }^{1 \#}$, Jianfei Shen ${ }^{2 \#}$, Liping Liu ${ }^{3 \#}$, Lanlan Shan ${ }^{4 \#}$, Jiaxi $\mathrm{He}^{1}$, Qihua $\mathrm{He}^{1}$, Long Jiang ${ }^{1}$, \\ Minzhang Guo ${ }^{1}$, Xuewei Chen ${ }^{1}$, Hui Pan ${ }^{1}$, Guilin Peng ${ }^{1}$, Honghui Shi ${ }^{1}$, Limin Ou ${ }^{3}$, Wenhua Liang ${ }^{1}$, \\ Jianxing $\mathbf{H e}^{1}$
}

${ }^{1}$ Department of Thoracic Surgery, the First Affiliated Hospital of Guangzhou Medical University, Guangzhou 510120, China; ${ }^{2}$ Department of Thoracic Surgery, Taizhou Hospital of Zhejiang Province, Wenzhou Medical University, Taizhou 317000, China; ${ }^{3}$ The Translational Medicine Laboratory, State Key Laboratory of Respiratory Disease, the First Affiliated Hospital of Guangzhou Medical University, Guangzhou 510120, China; ${ }^{4}$ Department of Health Management, Nanfang Hospital, Southern Medical University, Guangzhou 510120, China

Contributions: (I) Conception and design: Y Liu, J Shen, L Liu, L Shan, W Liang, J He; (II) Administrative support: None; (III) Provision of study materials or patients: None; (IV) Collection and assembly of data: Y Liu, J Shen, L Liu, L Shan, J He, Q He, L Jiang, M Zhang, X Chen, H Pan, G Peng, H Shi, L Ou; (V) Data analysis and interpretation: Y Liu, J Shen, L Liu, L Shan, W Liang, J He; (VI) Manuscript writing: All authors; (VII) Final approval of manuscript: All authors.

\#These authors contributed equally to the work.

Correspondence to: Jianxing He, MD, PhD, FACS. Department of Thoracic Surgery, the First Affiliated Hospital of Guangzhou Medical University, No. 151, Yanjiang Rd, Guangzhou 510120, China. Email: drjianxing.he@gmail.com.

Background: The correlation between the number of examined lymph nodes (ELNs) and lung cancerspecific survival (LCSS) of stage IA non-small cell lung cancer (NSCLC) patients, who underwent sublobar resection in which lymph node (LN) sampling was relatively restricted as compared with standard lobectomy remains unclear.

Methods: Patients from the Surveillance, Epidemiology, and End Results database with stage IA NSCLC who underwent sublobar resection were categorized based on ELN count (1-6 vs. $\geq 7$; the cut point 7 was identified by Cox model).

Results: Collectively, 3,219 patients with a median follow-up time of 37 months were included in this study (G1: 1-6 ELN, n=2,410; G2: $\geq 7$ ELN, n=809). The 5-year LCSS rate of the G1 and G2 cohorts were 75\% and $83 \%$, respectively. Cox analysis suggested that the LCSS of G1 cohort patients was lower as compared with the G2 cohort [hazard ratio $(\mathrm{HR})=1.530$; 95\% confidence interval $(\mathrm{CI}): 1.240-1.988, \mathrm{P}<0.001)$. Propensity score analysis also showed decreased survival of the matched G1 cohort (HR =1.499; $95 \%$ CI: 1.176-1.911; $\mathrm{P}=0.001)$.

Conclusions: The data suggested the ELNs $\leq 6$ were associated with poor prognoses. Adequate LN sampling is essential even for stage IA NSCLC patients undergoing sublobar resection.

Keywords: Lymph nodes; sublobar resection; stage IA; lung cancer; survival

Submitted May 07, 2018. Accepted for publication Nov 08, 2018.

doi: $10.21037 /$ jtd.2018.11.49

View this article at: http://dx.doi.org/10.21037/jtd.2018.11.49 


\section{Introduction}

Lung cancer is the leading cause of cancer-related deaths worldwide, with non-small cell lung cancer (NSCLC) accounting for nearly $85 \%$ of all diagnosed cases $(1,2)$. Approximately $25 \%$ of NSCLC patients are diagnosed in the early stages of the disease. Surgical resection is the best option for NSCLC patients. The 5-year overall survival (OS) rate of resected stage I NSCLC patients ranges from $55 \%$ to $80 \%(3,4)$.

For these patients, lymph node (LN) recurrence and metastasis may be the principal reason for unsatisfactory prognoses of NSCLC. Positive LN disease was undetected because of inadequate LN sampling and the shortage of LN dissection $(5,6)$. Smeltzer et al. in 2016 reported that the intrapulmonary metastasis $\mathrm{LN}$ discarded inadvertently from lung cancer resection specimens was associated with reduced survival (7). The accuracy of node staging and the number of positive $\mathrm{LN}$ are a strong prognostic indicator (8-10). LN involvement is one of the most important determinants of prognosis and treatment strategy decisions $(11,12)$.

Survival of stage IA NSCLC patients undergoing lobectomy could be improved by increasing the number of ELNs (13). However, these recommendations and potentially beneficial outcomes are based mainly on patients undergoing lobectomy, not sublobar resection. The current National Comprehensive Cancer Network (NCCN) guidelines recommend that sublobar resection should be based on sampling appropriate N1 and N2 LN stations, not on the number of ELN. The impact of ELNs on survival remains unclear and the evidence in the international guidelines for patients with stage IA NSCLC undergoing sublobar resection was based on very limited data.

In 2016, Speicher et al. reported better prognoses for stage IA NSCLC patients who underwent sublobar resection with nodal sampling (14). However, they did not elucidate the importance of the number of ELN. The significance of ELN count on survival of stage IA NSCLC patients undergoing sublobar resection has not yet been established. Herein, we investigated the correlation between the number of ELNs and LCSS in patients with stage IA NSCLC who underwent sublobar resection.

\section{Methods}

This study was approved by institutional board of the First Affiliated Hospital of Guangzhou Medical University.
The study used the Surveillance, Epidemiology, and End Results (SEER) database which includes patient demographics, cancer diagnoses and treatment related information, and cause of death. SEER is representative of the US population, with patient-level data abstracted from 18 geographically diverse populations that represent rural, urban, and regional populations (15). We identified patients aged $\geq 20$ years who underwent sublobar (wedge or segmental) resection between the years 2000 and 2013 with the following criteria: (I) pathologically confirmed diagnosis of stage IA (T1N0M0) NSCLC (based on the 8th edition of the Union for International Cancer Control/American Joint Committee on Cancer Tumor, Node, and Metastasis (TNM) classification for lung cancer); (II) first primary NSCLC treated by sublobar resection; and (III) at least one LN examined. Patients with missing ELN count values and clinical features were excluded from the study.

The Cox proportional hazards regression model was used to determine the effect of ELN count on LCSS, which were adjusted for other significant prognostic factors (age at diagnosis, sex, race, histology, grade, tumor size, and surgery type). Patients were stratified based on the per ELN count, ranging from 1 to 12. Finally, a cut point of 7 was identified based on the maximum hazard ratio (HR) and the minimum $\mathrm{P}$ value, and the structural break points were considered the threshold of clinical impact (16). Complete analyses of the ELN count on LCSS are shown in Table 1. Patients were divided into 2 groups: G1 (1-6 ELNs) and G2 ( $\geq 7 \mathrm{ELNs})$.

We performed propensity score matching (PSM) analysis to compare survival among the G1 and G2 cohorts. In each analysis, a propensity score for the G2 cohort was created using logistic regression based on potential confounding variables, including age, sex, race, tumor site, histology, grade, tumor size and surgery type. Patient characteristics were compared with t-tests for continuous variables and $\chi^{2}$ tests for categorical variables. Survival was calculated from date of diagnosis until date of death or date of last followup (December 31, 2015). When analyzing the LCSS, deaths from other causes were censored at the date of death. LCSS were estimated by the Kaplan-Meier method and the logrank test was used to assess differences.

Univariate and multivariate analyses were performed using Cox proportional hazards models to calculate adjusted HR and their $95 \%$ confidence interval (CI) relating to the variables as described. All data were analyzed using SPSS 22.0 or Prism 5.0 software. Results were considered to be statistically significant when $\mathrm{P}<0.05$. 
Table 1 The effect of examined lymph node (ELN) on lung cancer-specific survival as determined by the Cox proportional hazards regression model.

\begin{tabular}{lcc}
\hline ELN group (Number of patients) & Hazard ratio & 95\% confidence interval \\
\hline $1(n=678)$ vs. $2-63(n=2,541)$ & 1.326 & $1.107-1.588$ \\
$1-2(n=1,236)$ vs. $3-63(\mathrm{n}=1,983)$ & 1.340 & $1.139-1.576$ \\
$1-3(\mathrm{n}=1,676)$ vs. $4-63(\mathrm{n}=1,543)$ & 1.397 & $1.183-1.650$ \\
$1-4(\mathrm{n}=1,986)$ vs. $5-63(\mathrm{n}=1,233)$ & 1.355 & $1.137-1.615$ \\
$1-5(\mathrm{n}=2,215)$ vs. $6-63(\mathrm{n}=1,004)$ & 1.481 & $1.222-1.794$ \\
$1-6(\mathrm{n}=2,410)$ vs. $7-63(\mathrm{n}=809)$ & 1.520 & $1.232-1.876$ \\
$1-7(\mathrm{n}=2,564)$ vs. $8-63(\mathrm{n}=655)$ & 1.433 & $1.142-1.798$ \\
$1-8(\mathrm{n}=2,669)$ vs. $9-63(\mathrm{n}=550)$ & 1.304 & $1.031-1.649$ \\
$1-9(\mathrm{n}=2,757)$ vs. $10-63(\mathrm{n}=462)$ & 1.323 & $1.026-1.708$ \\
$1-10(\mathrm{n}=2,829)$ vs. $11-63(\mathrm{n}=390)$ & 1.212 & 0.001 \\
$1-11(\mathrm{n}=2,889)$ vs. $12-63(\mathrm{n}=330)$ & 1.198 & $0.026-1.587$ \\
$1-12(\mathrm{n}=2,938)$ vs. $13-63(\mathrm{n}=281)$ & 1.241 & 0.001 \\
\hline
\end{tabular}

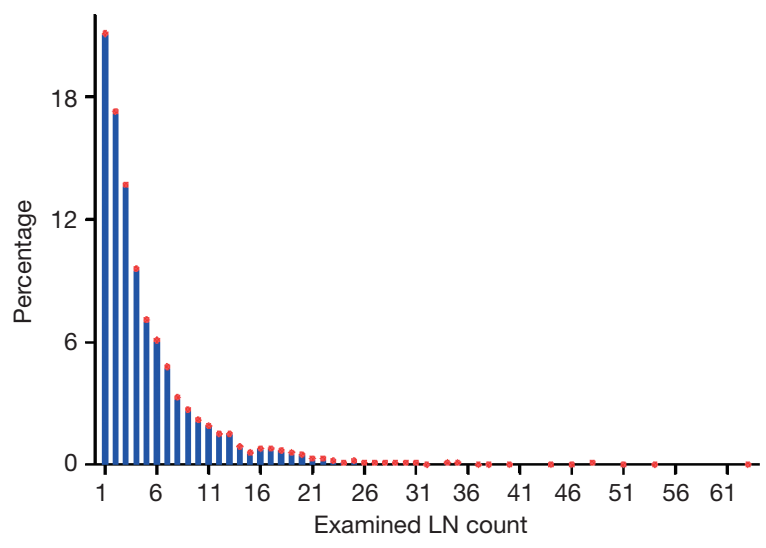

Figure 1 Distribution of the number of examined lymph nodes (ELNs) in the study cohort. Each red dots represents a number, and the height of bar represents the proportion of the whole.

\section{Results}

As a result of selection criteria, a total of 3,219 patients with pathologic stage IA (T1NOM0) NSCLC were included. The G1 cohort (2,410 patients) underwent sublobar resection with 1-6 ELNs. The median age within the cohort was 69 years (range, 20-92 years), and the median tumor size was $1.6 \mathrm{~cm}$ (range, $1-3.0 \mathrm{~cm}$ ). The distribution of ELN number in this study cohort is shown in Figure 1 (median, 3 ; interquartile range, 2-7). Patients with $\geq 7$ ELNs were more likely to be younger $(\mathrm{P}=0.007)$, adenoid differentiation $(\mathrm{P}<0.001)$, grade $\mathrm{I}-\mathrm{II}$ disease $(\mathrm{P}=0.001)$ and to have a segmental resection $(\mathrm{P}=0.002)$, but there were no significant differences between the G1 and G2 cohorts in sex, race, tumor site and size ( $\mathrm{P}>0.05$ for all comparisons). However, after PSM, the differences between groups were well balanced (Figures S1-S3). The baseline characteristics of the full cohort and patients in the G1 and G2 cohorts are presented in Table 2 .

The 5-year LCSS rates of patients in the G1 and G2 cohorts were $75 \%$ and $83 \%$, respectively. Cox analyses showed that the LCSS of patients in the G1 cohort was inferior as compared to the $\mathrm{G} 2$ cohort $(\mathrm{HR}=1.530 ; 95 \% \mathrm{CI}$ : 1.240-1.988; $\mathrm{P}<0.001$; Figure $2 A$ ). $\mathrm{PSM}$ analyses also showed decreased survival of the matched G1 cohort patients (HR $=1.499 ; 95 \%$ CI: 1.176-1.911; $\mathrm{P}=0.001$; Figure 2B).

Based on subset analysis of patients with wedge resection, the 5-year LCSS rates of the G1 and G2 cohorts were $74 \%$ and $81 \%$, respectively. Survival of G1 cohort patients with wedge resection was poorer as compared to G2 (HR $=1.433 ;$ 95\% CI: 1.153-1.780; P=0.001). For patients with segmental resection, the 5 -year LCSS rates of the G1 and G2 cohorts were $78 \%$ and $87 \%$, respectively. Cox analysis showed decreased survival of G1 patients with segmental resection as compared to the G2 cohort (HR $=1.514 ; 95 \%$ CI: $1.048-2.187 ; \mathrm{P}=0.027)$. Results of PSM analysis were similar. The Kaplan-Meier plots for the subsets of patients with wedge and segmental resection are presented in Figures 3,4. 
Table 2 The baseline characteristics of patients in the study cohort

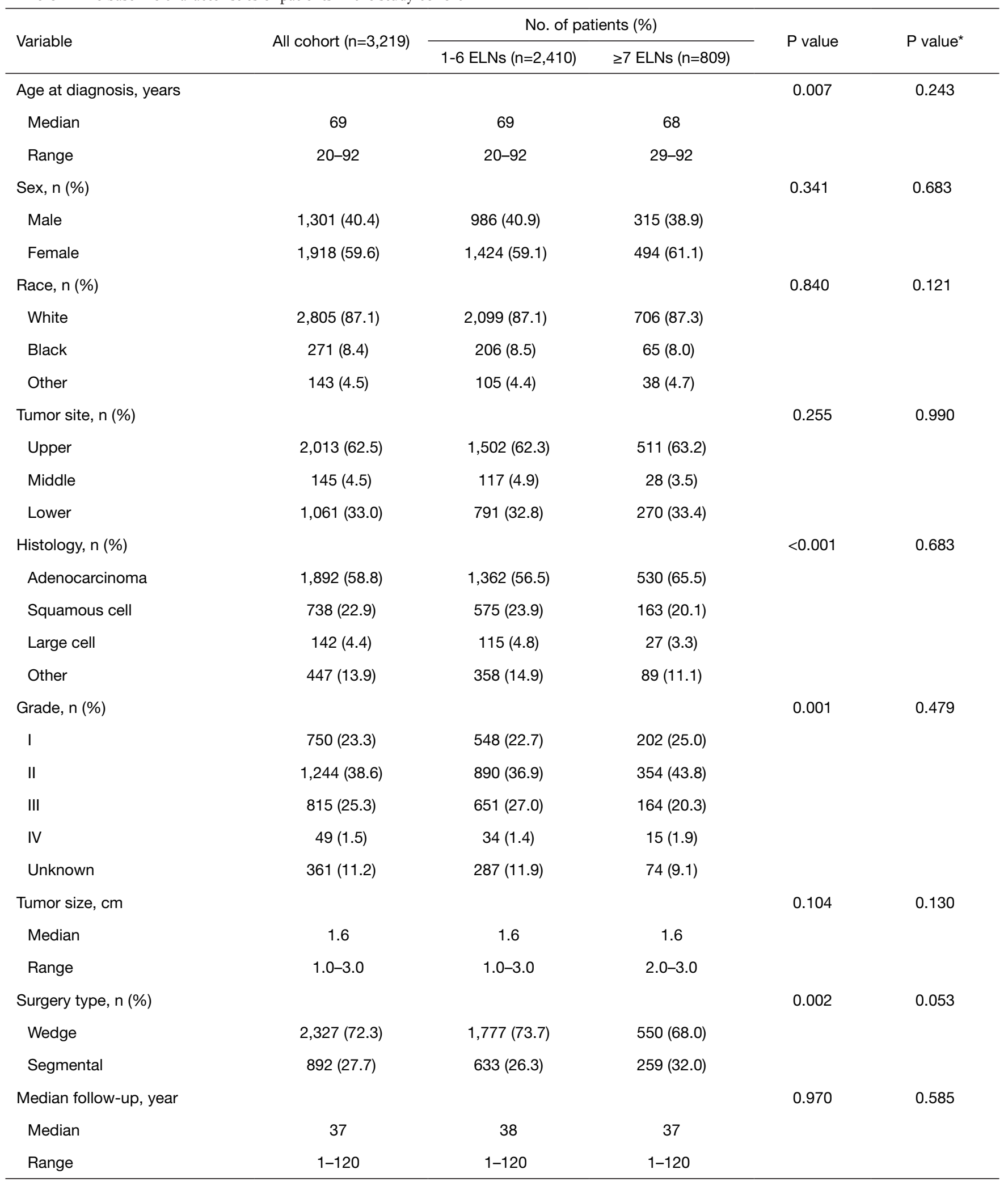

*, $\mathrm{P}$ value was adjusted by propensity score matching analysis. ELN, examined lymph node. 

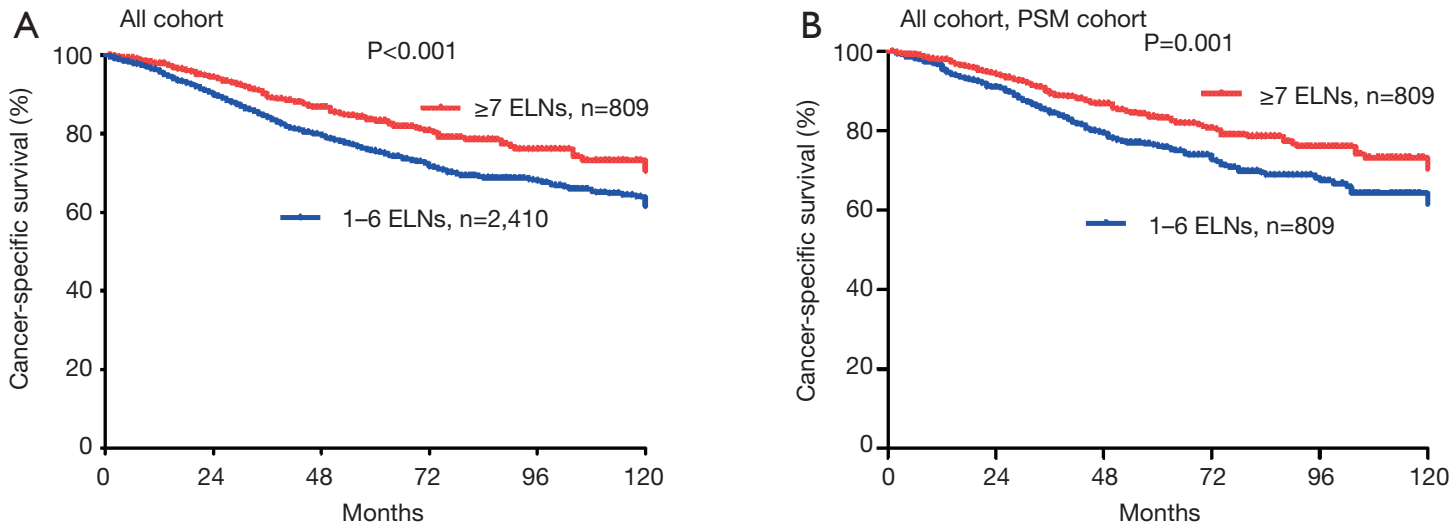

Figure 2 Kaplan-Meier plot for lung cancer-specific survival in the study cohort before (A) and after (B) propensity score matched.
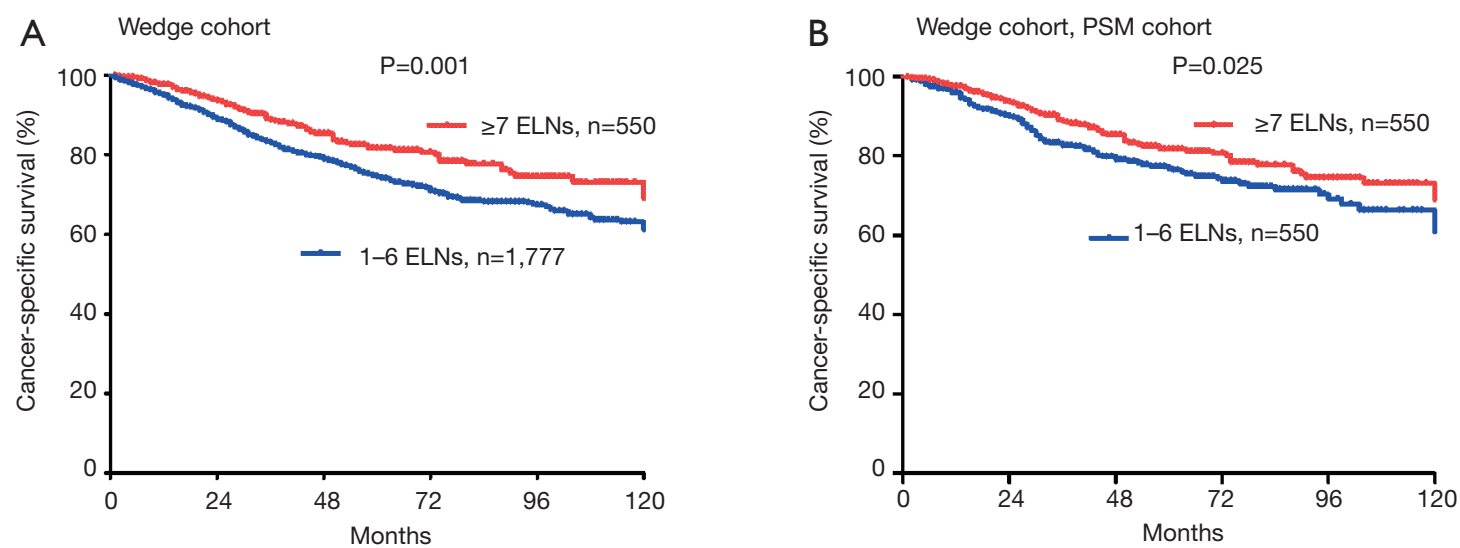

Figure 3 Kaplan-Meier plot for lung cancer-specific survival in patients with stage IA NSCLC undergoing wedge resection before (A) and after (B) propensity score matched.
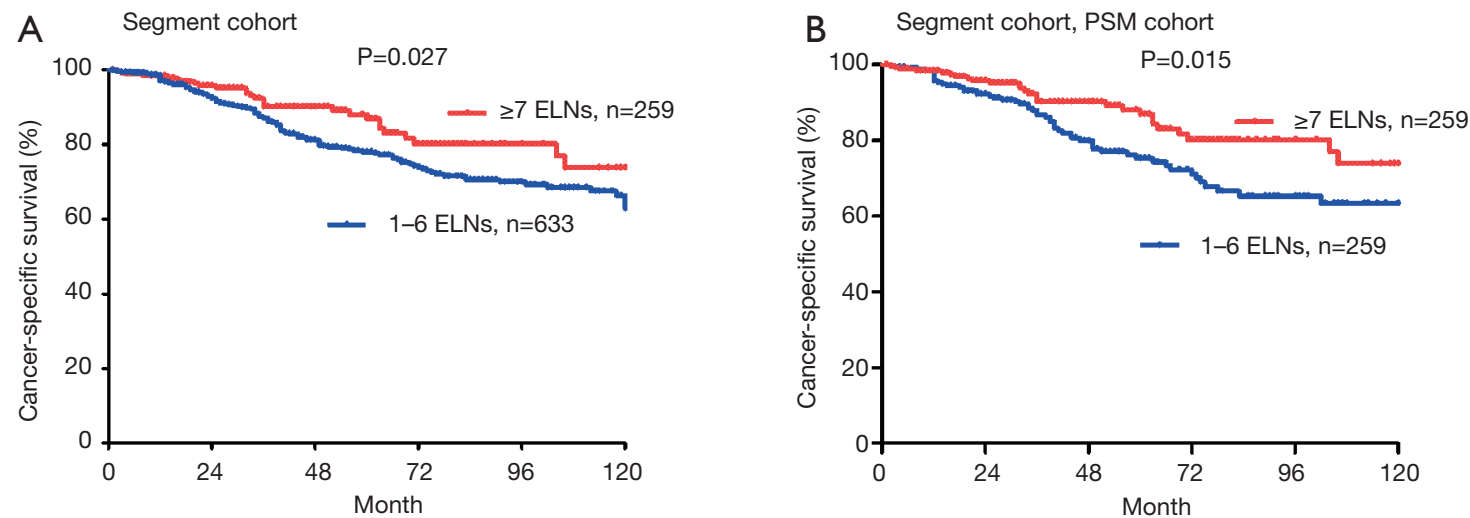

Figure 4 Kaplan-Meier plot for lung cancer-specific survival in patients with stage IA NSCLC undergoing segmental resection before (A) and after (B) propensity score matched. 
On univariate analysis, the Cox analyses indicated that 1-6 ELNs did not improve LCSS. Other adverse prognostic factors were older age, male sex, larger grade disease and wedge resection. Race and tumor site were not prognostic. Based on multivariate analysis of all patients, older age ( $\mathrm{HR}=1.029 ; 95 \% \mathrm{CI}: 1.019-1.038 ; \mathrm{P}<0.001)$, and larger grade disease $(\mathrm{HR}=2.063 ; 95 \% \mathrm{CI}: 1.564-2.720 ; \mathrm{P}<0.001)$ had a negative impact on OS. Females (HR $=0.677 ; 95 \%$ CI: $0.575-0.798 ; \mathrm{P}<0.001)$, segmental resection $(\mathrm{HR}=0.768$; 95\% CI: 0.636-0.928; $\mathrm{P}=0.006)$ and $\geq 7$ ELNs $(\mathrm{HR}=0.691$; 95\% CI: $0.559-0.855 ; \mathrm{P}=0.001)$ were significantly associated with improved LCSS. The results of all univariate and multivariate analyses are listed in Table 3.

\section{Discussion}

Results of this study suggest that stage IA NSCLC patients who underwent sublobar resection with $\leq 6$ ELNs were associated with a poorer LCSS as compared to patients with $\geq 7$ ELNs. We obtained consistent results in the subset analysis for patients with wedge and segmental resection. These results were confirmed by the Cox model and PSM analyses.

It is assumed that more ELNs provide a better opportunity to identify positive LNs; that is, a larger number of ELNs may reduce the risk of undetected positive LNs and may allow for improved outcomes. These findings may be attributed to more thorough examinations, the removal of potential remnants, and proper delivery of adjuvant chemotherapy to improve long-term survival.

There are limited data in the existing NCCN guidelines that address the impact of ELN counts on patients undergoing segmental or wedge resection; thus, surgical sampling of ELN counts of surgical specimens are not uniformly performed. The number of ELNs dissected by sublobar resection often varies between surgeons and pathologists. Therefore, survival of most patients with an inadequate ELN count is poor. Our results suggested that stage IA NSCLC patients undergoing sublobar resection with $\geq 7$ ELNs correlated with improved prognoses. Previous studies suggested that the ELNs combined with tumor size or the ratios of positive LNs could be an independent prognostic factor and a reasonable stratification criterion in patients with pathologic node-negative NSCLC $(17,18)$.

Stiles et al. in 2017 reported that $\mathrm{LN}$ removal decreased locoregional recurrence $(\mathrm{P}=0.024)$ and increased the 5 -year survival rate $(\mathrm{P}=0.025)$, and $\mathrm{LN}$ sampling did not add to morbidity and did not increase the length of stay (19). Varlotto et al. in 2009 showed that patients with dissected lymph nodes were associated with an increase in the 5-year OS rate (from $42 \%$ to $58 \%$ ) and in DFS (from $58 \%$ to $73 \%$ ) as compared to patients with no lymphadenectomy (20). Additionally, several previous studies support the viewpoint that the number of counted ELNs is beneficial for patients with declared node-negative disease $(5,6,13,16,18,21,22)$.

However, other groups believe that recommending an optimal number of nodes is not an appropriate prognostic factor for stage I NSCLC patients $(23,24)$. Altorki et al. found that LN sampling was more commonly performed on patients undergoing segmental resection as compared to patients undergoing wedge resection, with more stations sampled and more nodes resected in segmental resection patients. There were no differences between wedge and segmental resection in local recurrence $(\mathrm{P}=0.680)$ or 5 -year disease-free survival (DFS: $51 \%$ vs. 53\%, $\mathrm{P}=0.700$ ) (25). Additionally, the results of the ACOSOG Z0030 trial showed that if systematic and thorough resection sampling of the mediastinal and hilar nodes is negative, mediastinal LN dissection does not improve survival of patients with early NSCLC (26). Izbicki et al. also reported that radical systematic mediastinal LN dissection did not influence the DFS or OS for NSCLC patients without overt LN involvement (27).

This study has some limitations. Bias is inevitable due to the retrospective nature. Some important information is missing, such as the access to perform limited resection (open or VATS) and the surgical technique of participating surgeons. We were not able to investigate other important points such as the impact of the number of LNs in the N1 and N2 (hilar and mediastinal) stations on our data analysis. In addition, two possible causes could have resulted in a miscount of ELN: underestimation as a result of the difficulty in separating each LN in the dissected tissues, and overestimation because of fragmentation of nodal tissue during the removal of LN. The SEER does not provide information on clinical stage, performance status, smoking, preoperative cardiopulmonary function, postoperative complications, or length of stay.

\section{Conclusions}

In summary, our data indicates that $\leq 6$ ELNs was associated with poor prognosis of patients with pathological stage IA NSCLC undergoing sublobar resection. Adequate LN examination is essential even in patients with pathological 
Table 3 Univariate and multivariate analysis on lung cancer-specific survival of the study cohort

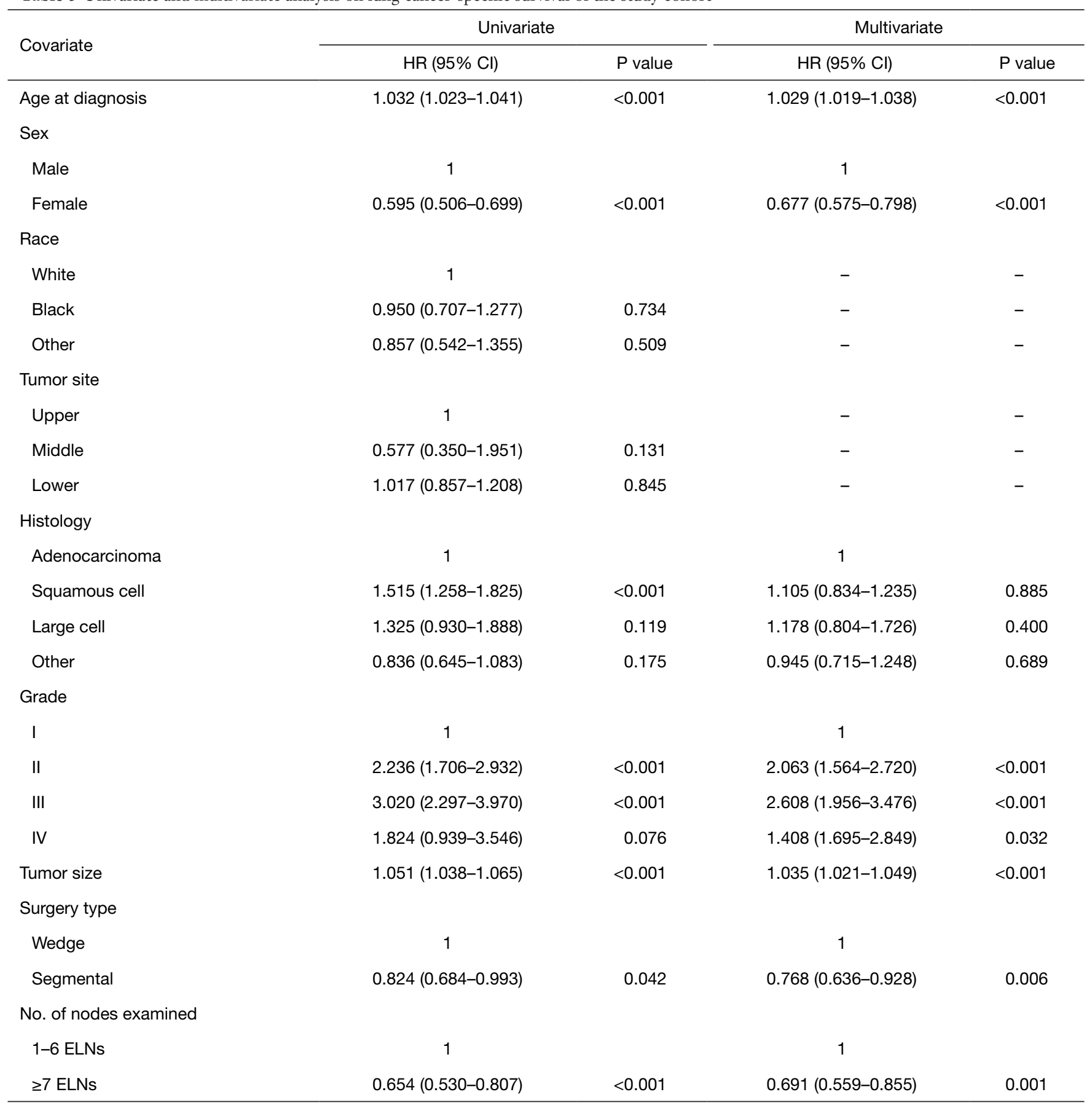


stage IA NSCLC.

\section{Acknowledgements}

We acknowledge the efforts of the SEER program tumor registries in the creation of the SEER database. The authors thank Ms. Lindsey Hamblin for her help in editing the manuscript.

\section{Footnote}

Conflicts of Interest: The authors have no conflicts of interest to declare.

Ethical Statement: This study was approved by institutional board of the First Affiliated Hospital of Guangzhou Medical University.

\section{References}

1. Chen $\mathrm{W}$, Zheng R, Baade PD, et al. Cancer statistics in China, 2015. CA Cancer J Clin 2016;66:115-32.

2. Siegel RL, Miller KD, Jemal A. Cancer statistics, 2016. CA Cancer J Clin 2016;66:7-30.

3. Soysal O, Walsh GL, Nesbitt JC, et al. Resection of sternal tumors: extent, reconstruction, and survival. Ann Thorac Surg 1995;60:1353-8; discussion 1358-9.

4. Harpole DH Jr, Herndon JE 2nd, Young WG Jr, et al. Stage I non-small cell lung cancer. A multivariate analysis of treatment methods and patterns of recurrence. Cancer 1995;76:787-96.

5. Xu F, Qi L, Yue D, et al. The effect of the extent of lymph node dissection for stage IA non-small-cell lung cancer on patient disease-free survival. Clin Lung Cancer 2013;14:181-7.

6. Gajra A, Newman N, Gamble GP, et al. Effect of number of lymph nodes sampled on outcome in patients with stage I non-small-cell lung cancer. J Clin Oncol 2003;21:1029-34.

7. Smeltzer MP, Faris N, Yu X, et al. Missed Intrapulmonary Lymph Node Metastasis and Survival After Resection of Non-Small Cell Lung Cancer. Ann Thorac Surg 2016;102:448-53.

8. Rusch VW, Crowley J, Giroux DJ, et al. The IASLC Lung Cancer Staging Project: proposals for the revision of the $\mathrm{N}$ descriptors in the forthcoming seventh edition of the TNM classification for lung cancer. J Thorac Oncol 2007;2:603-12.
9. Fukui T, Mori S, Yokoi K, et al. Significance of the number of positive lymph nodes in resected non-small cell lung cancer. J Thorac Oncol 2006;1:120-5.

10. Lee GD, Kim DK, Moon DH, et al. A comparison of the proposed classifications for the revision of $\mathrm{N}$ descriptors for non-small-cell lung cancer. Eur J Cardiothorac Surg 2016;49:580-8.

11. American Cancer Society. Small cell lung cancer, (2016). Available online: http://www.cancer.org/acs/groups/cid/ documents/webcontent/003116-pdf.pdf. Accessed June 16, 2016.

12. Liang W, Zhang L, Jiang G, et al. Development and validation of a nomogram for predicting survival in patients with resected non-small-cell lung cancer. J Clin Oncol 2015;33:861-9.

13. Ou SH, Zell JA. Prognostic significance of the number of lymph nodes removed at lobectomy in stage IA non-small cell lung cancer. J Thorac Oncol 2008;3:880-6.

14. Speicher PJ, Gu L, Gulack BC, et al. Sublobar Resection for Clinical Stage IA Non-small-cell Lung Cancer in the United States. Clin Lung Cancer 2016;17:47-55.

15. National Cancer Institute. Surveillance, Epidemiology, and End Results (SEER) program. SEER*Stat Database. Available online: http://www.seer.cancer.gov

16. Liang W, He J, Shen Y, et al. Impact of Examined Lymph Node Count on Precise Staging and Long-Term Survival of Resected Non-Small-Cell Lung Cancer: A Population Study of the US SEER Database and a Chinese MultiInstitutional Registry. J Clin Oncol 2017;35:1162-70.

17. Nwogu CE, Groman A, Fahey D, et al. Number of lymph nodes and metastatic lymph node ratio are associated with survival in lung cancer. Ann Thorac Surg 2012;93:1614-9; discussion 1619-20.

18. Yang $M$, Cao H, Guo $X$, et al. The number of resected lymph nodes (nLNs) combined with tumor size as a prognostic factor in patients with pathologic N0 and $\mathrm{Nx}$ non-small cell lung cancer. PLoS One 2013;8:e73220.

19. Stiles BM, Kamel MK, Nasar A, et al. The importance of lymph node dissection accompanying wedge resection for clinical stage IA lung cancer dagger. Eur J Cardiothorac Surg 2017;51:511-7.

20. Varlotto JM, Recht A, Nikolov M, et al. Extent of lymphadenectomy and outcome for patients with stage I nonsmall cell lung cancer. Cancer 2009;115:851-8.

21. Zhou H, Tapias LF, Gaissert HA, et al. Lymph Node Assessment and Impact on Survival in Video-Assisted Thoracoscopic Lobectomy or Segmentectomy. Ann Thorac Surg 2015;100:910-6. 
22. Samayoa AX, Pezzi TA, Pezzi CM, et al. Rationale for a Minimum Number of Lymph Nodes Removed with Non-Small Cell Lung Cancer Resection: Correlating the Number of Nodes Removed with Survival in 98,970 Patients. Ann Surg Oncol 2016;23:1005-11.

23. Riquet M, Legras A, Mordant P, et al. Number of mediastinal lymph nodes in non-small cell lung cancer: a Gaussian curve, not a prognostic factor. Ann Thorac Surg 2014;98:224-31.

24. Gulack BC, Yang CF, Speicher PJ, et al. The impact of tumor size on the association of the extent of lymph node resection and survival in clinical stage I non-small cell lung cancer. Lung Cancer 2015;90:554-60.

25. Altorki NK, Kamel MK, Narula N, et al. Anatomical segmentectomy and wedge resections are associated with comparable outcomes for small cT1N0 non-small cell lung cancer. J Thorac Oncol 2016;11:1984-92.

26. Darling GE, Allen MS, Decker PA, et al. Randomized trial of mediastinal lymph node sampling versus complete lymphadenectomy during pulmonary resection in the patient with N0 or N1 (less than hilar) non-small cell carcinoma: results of the American College of Surgery Oncology Group Z0030 Trial. J Thorac Cardiovasc Surg 2011;141:662-70.

27. Izbicki JR, Passlick B, Pantel K, et al. Effectiveness of radical systematic mediastinal lymphadenectomy in patients with resectable non-small cell lung cancer: results of a prospective randomized trial. Ann Surg 1998;227:138-44.
Cite this article as: Liu Y, Shen J, Liu L, Shan L, He J, He Q, Jiang L, Guo M, Chen X, Pan H, Peng G, Shi H, Ou L, Liang W, He J. Impact of examined lymph node counts on survival of patients with stage IA non-small cell lung cancer undergoing sublobar resection. J Thorac Dis 2018;10(12):6569-6577. doi: 10.21037/jtd.2018.11.49 
Sample sizes

\begin{tabular}{|c|c|c|c|c|c|c|c|c|}
\hline \multirow{2}{*}{ Subsamples } & \multicolumn{2}{|c|}{ All } & \multicolumn{2}{c|}{ Matched } & \multicolumn{2}{c|}{ Unmatched } & \multicolumn{2}{c|}{ Discarded } \\
\cline { 2 - 9 } & Control & Treated & Control & Treated & Control & Treated & Control & Treated \\
\hline (all cases) & 2,410 & 809 & 809 & 809 & 1,601 & 0 & 0 & 0 \\
\hline
\end{tabular}

Relative multivariate imbalance L1 (lacus, King, \& Porro, 2010)

\begin{tabular}{|c|c|c|}
\hline & Before matching & After matching \\
\hline (all cases) & 0.931 & 0.862 \\
\hline
\end{tabular}

Distribution of propensity scores

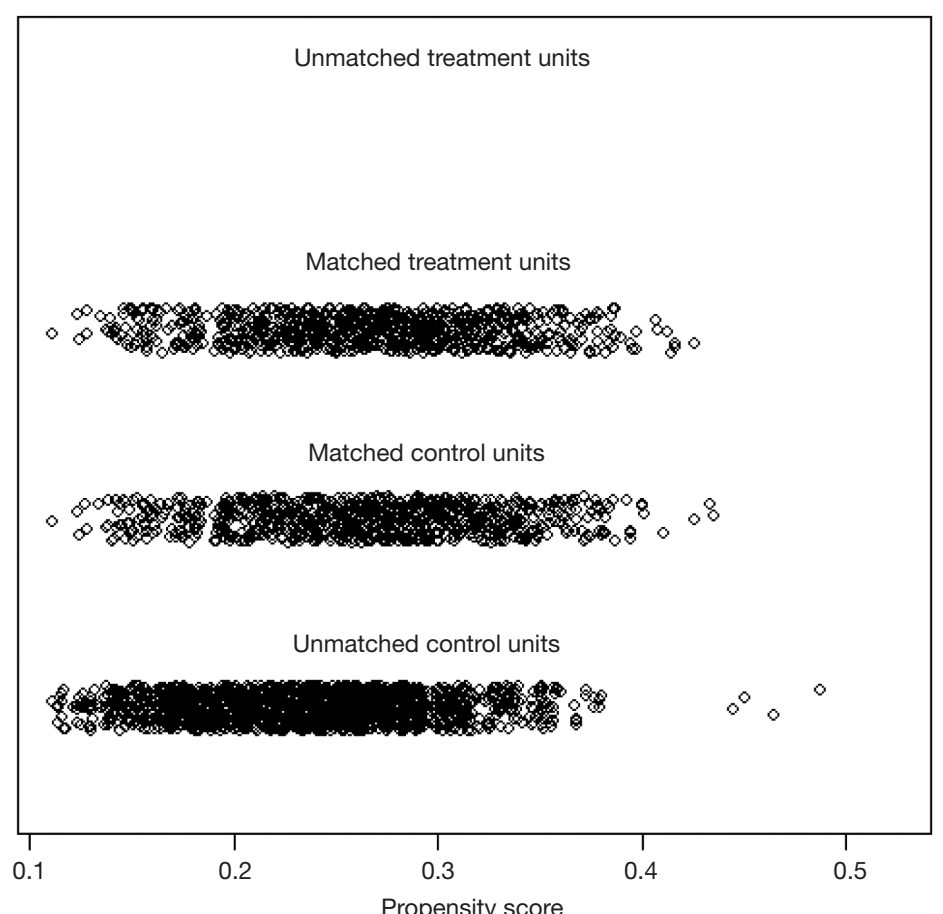

Unmatched Treated

Matched Treated
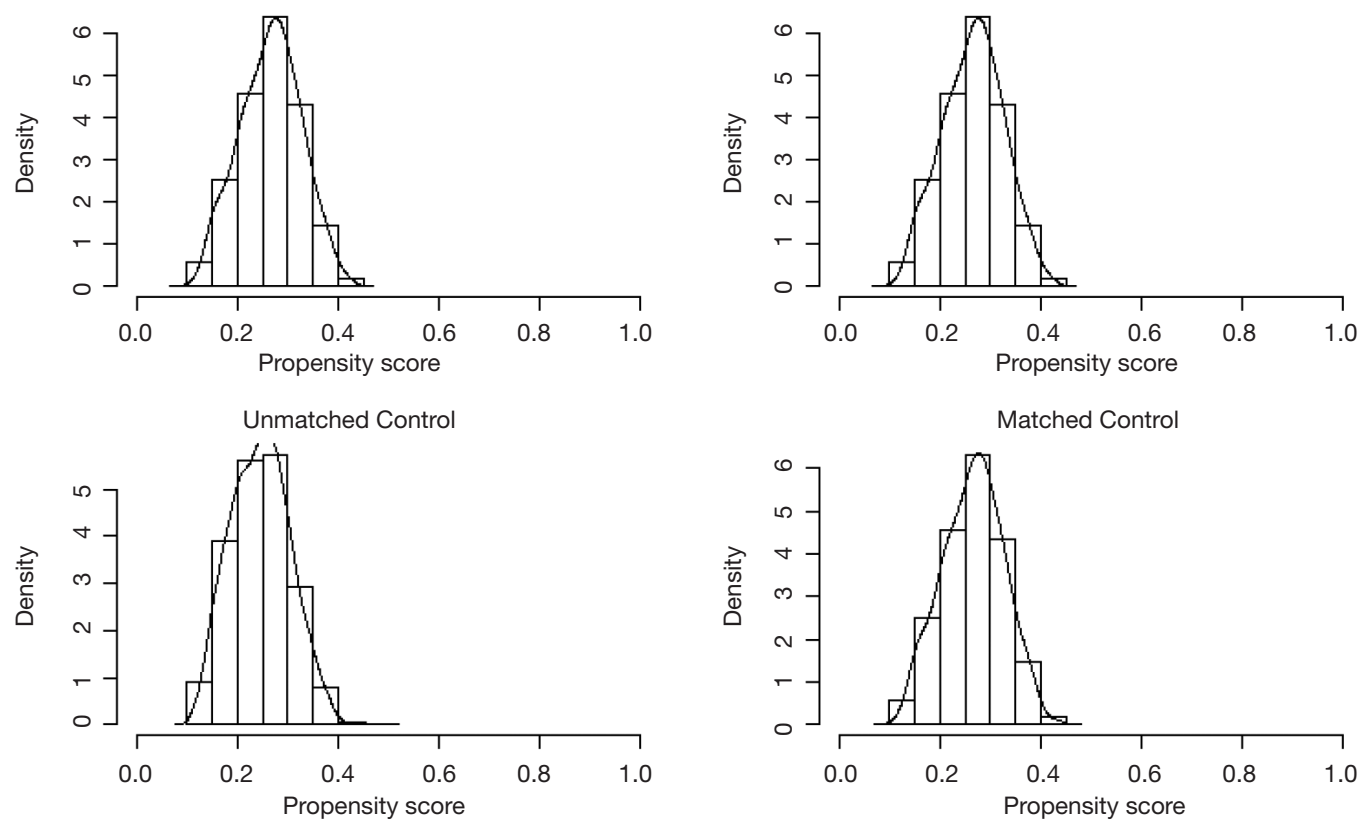

Figure S1 The charts of propensity score matching in the all study cohort. 
Sample sizes

\begin{tabular}{|c|c|c|c|c|c|c|c|c|}
\hline \multirow{2}{*}{ Subsamples } & \multicolumn{2}{|c|}{ All } & \multicolumn{2}{c|}{ Matched } & \multicolumn{2}{c|}{ Unmatched } & \multicolumn{2}{c|}{ Discarded } \\
\cline { 2 - 9 } & Control & Treated & Control & Treated & Control & Treated & Control & Treated \\
\hline (all cases) & 1,777 & 550 & 550 & 550 & 1,227 & 0 & 0 & 0 \\
\hline
\end{tabular}

Relative multivariate imbalance L1

(lacus, King, \& Porro, 2010)

\begin{tabular}{|l|c|c|}
\hline & Before matching & After matching \\
\hline (all cases) & 0.921 & 0.840 \\
\hline
\end{tabular}

Distribution of propensity scores

Unmatched treatment units

Matched treatment units

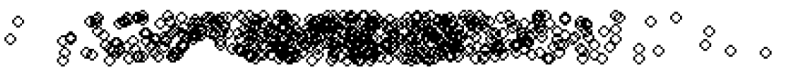

Matched control units

$\circ \quad 00 \% 8 \%$ \%

Unmatched control units

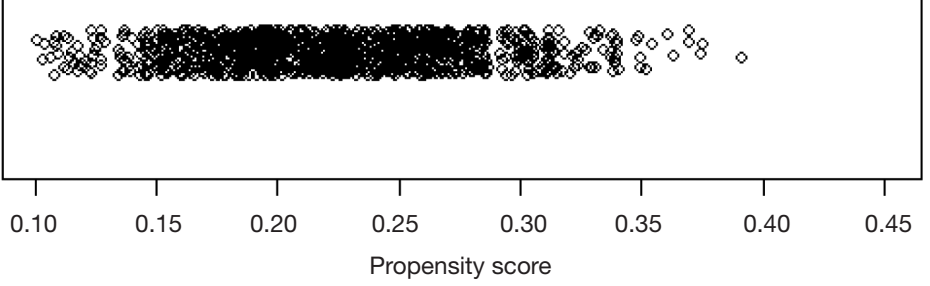

Unmatched Treated

Matched Treated
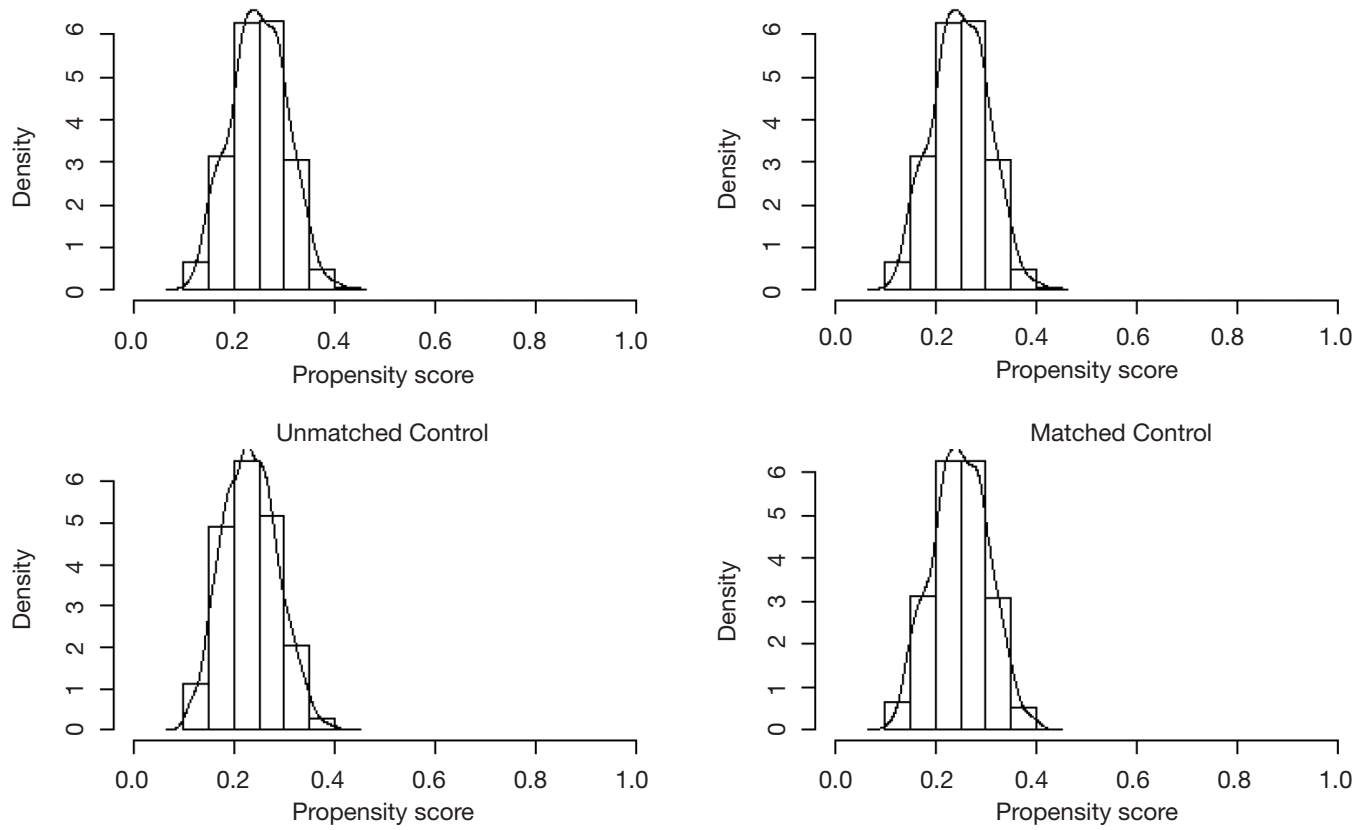

Figure S2 The charts of propensity score matching in the wedge cohort. 
Sample sizes

\begin{tabular}{|c|c|c|c|c|c|c|c|c|}
\hline \multirow{2}{*}{ Subsamples } & \multicolumn{2}{|c|}{ All } & \multicolumn{2}{c|}{ Matched } & \multicolumn{2}{c|}{ Unmatched } & \multicolumn{2}{c|}{ Discarded } \\
\cline { 2 - 9 } & Control & Treated & Control & Treated & Control & Treated & Control & Treated \\
\hline (all cases) & 633 & 259 & 259 & 259 & 374 & 0 & 0 & 0 \\
\hline
\end{tabular}

Relative multivariate imbalance L1

(lacus, King, \& Porro, 2010)

\begin{tabular}{|l|c|c|}
\hline & Before matching & After matching \\
\hline (all cases) & 0.897 & 0.865 \\
\hline
\end{tabular}

Distribution of propensity scores
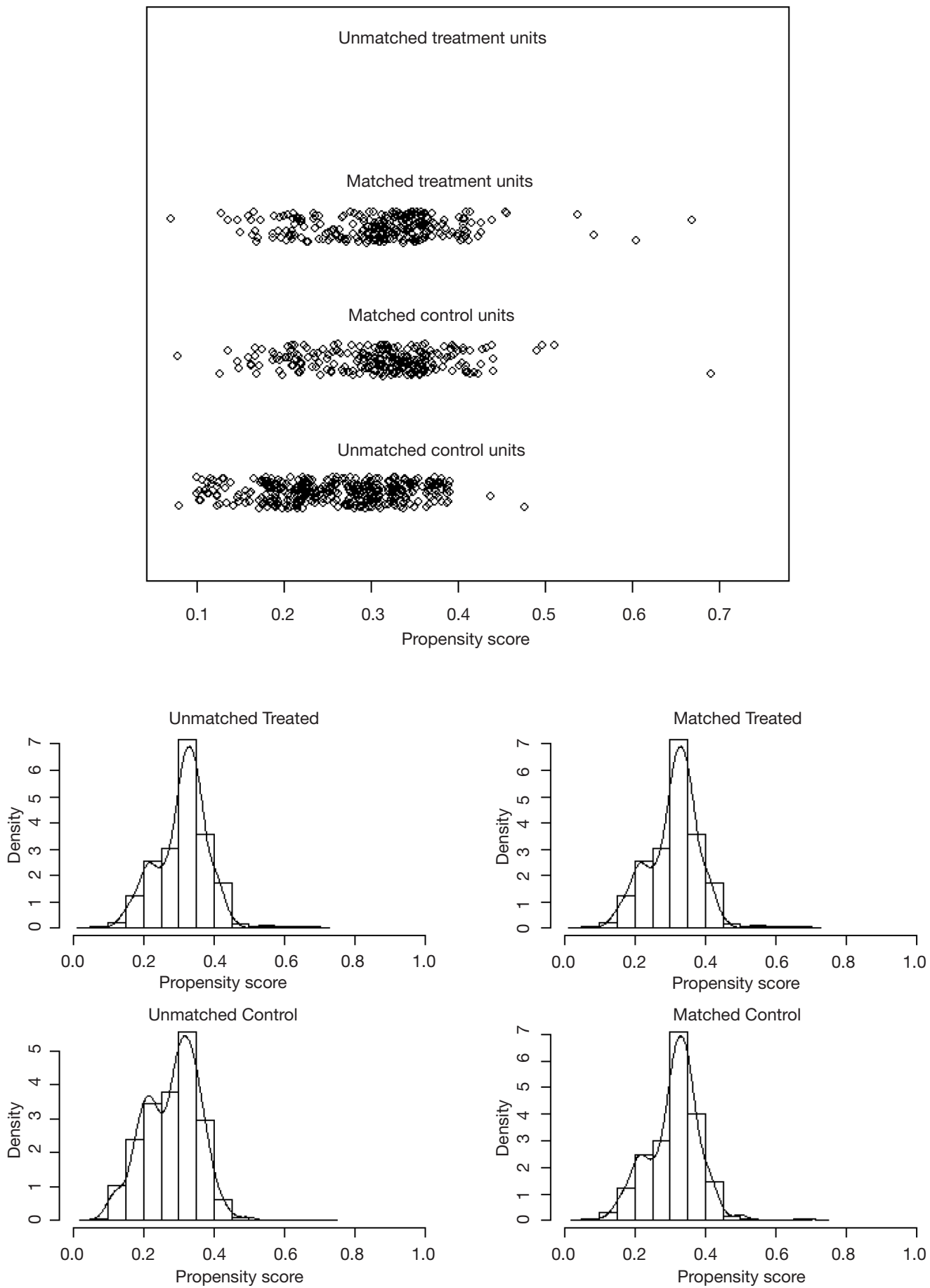

Figure S3 The charts of propensity score matching in the segment cohort. 